Raquel Quadros Velloso

\title{
Simulação Numérica de Problemas de Acoplamento Fluidomecânico em Meios Porosos Utilizando o Método dos Elementos Discretos
}

Tese apresentada ao Programa de Pós-graduação em Engenharia Civil da PUC-Rio como requisito parcial para obtenção do título de Doutor em Engenharia Civil

Orientador: Prof. Eurípedes do Amaral Vargas Jr. 


\title{
Raquel Quadros Velloso
}

\section{Simulação Numérica de Problemas de Acoplamento Fluidomecânico em Meios Porosos Utilizando o Método dos Elementos \\ Discretos}

Tese apresentada como requisito parcial para obtenção do título de Doutor pelo Programa de Pós-graduação em Engenharia Civil da PUC-Rio. Aprovada pela Comissção Examinadora abaixo assinada.

\author{
Prof. Eurípedes do Amaral Vargas Jr. \\ Orientador \\ Departamento de Engenharia Civil - PUC-Rio \\ Dr. Antônio Cláudio Soares \\ CENPES/Petrobras \\ Dr. Armando Prestes \\ Departamento de Engenharia Civil/PUC-Rio
}

Dr. Luis Carlos Baralho Bianco Chevron Energy Technology Company

Prof. Márcio da Silveira Carvalho

Departamento de Engenharia Mecânica/PUC-Rio

Prof. José Eugenio Leal

Coordenador Setorial do Centro Técnico Científico - PUC-Rio 
Todos os direitos reservados. É proibida a reprodução total ou parcial do trabalho sem autorização da universidade, do autor e do orientador.

Raquel Quadros Velloso

Ficha Catalográfica

Velloso, Raquel Q.

Simulação Numérica de Problemas de Acoplamento Fluidomecânico em Meios Porosos Utilizando o Método dos Elementos Discretos / Raquel Quadros Velloso; orientador: Eurípedes do Amaral Vargas Jr.. - 2010.

$94 \mathrm{f}$ : il. (color.) ; $30 \mathrm{~cm}$

1. Tese (doutorado) - Pontifícia Universidade Católica do Rio de Janeiro, Departamento de Engenharia Civil, 2010 Inclui bibliografia.

1. Engenharia Civil - Teses. 2. Método dos Elementos Discretos. 3. Método de Lattice-Boltzmann. 4. Dano Mecânico de Formação. 5. Produção de Sólidos. I. Vargas Jr., Eurípedes do Amaral. II. Pontifícia Universidade Católica do Rio de Janeiro. Departamento de Engenharia Civil. III. Título. 


\section{Agradecimentos}

Agradeço ao Vargas pela orientação, apoio e incentivo sem os quais eu não teria concluído este trabalho.

Agradeço à minha mãe que sempre faz tudo ficar mais fácil e ao meu pai por ter financiado meus estudos por tanto tempo.

Agradeço à Flavia, ao João, ao Eduardo, à Tatiana, ao Fábio, à Vanessa, ao Maurício, ao Bê, ao Bruno e à Tessa que compreenderam e suportaram minha preocupação e meu mau humor durante este longo período. E à tia Elisa que eu sei que mesmo de longe está sempre torcendo e rezando por mim.

Agradeço ao Antônio Claudio Soares que sempre incentivou meu trabalho desde antes do mestrado, ao Silvestre pelas conversas e por sempre ter (e emprestar) todos os livros que alguém precise, e ao Luis Arnaldo pelas discussões sobre o DEM.

Agradeço à Rita e à Fátima do Departamento de Engenharia Civil da PUC-Rio por terem sempre ajudado e resolvido, com simpatia e bom humor, meus problemas burocráticos na PUC-Rio.

Agradeço ao CNPq pelo suporte financeiro. 


\section{Resumo}

Velloso, Raquel Q.; Vargas Jr., Eurípedes do Amaral. Simulação Numérica de Problemas de Acoplamento Fluidomecânico em Meios Porosos Utilizando o Método dos Elementos Discretos. Rio de Janeiro, 2010. 94p. Tese de Doutorado - Departamento de Engenharia Civil, Pontifícia Universidade Católica do Rio de Janeiro.

Esta pesquisa é motivada, principalmente, por problemas da geomecânica do petróleo como produção de sólidos em poços produtores e dano mecânico de formação. Produção de sólidos é o fenômeno onde partículas sólidas são produzidas juntamente com os fluidos de um reservatório de formação geralmente pouco ou não consolidada, podendo também ocorrer em formações mais resistentes. Dano de formação é o termo usado para identificar a redução da permeabilidade por diversos processos que ocorrem nas formações geológicas, e que reduzem a produtividade e injetividade de poços de sistemas de produção de óleo e gás. Neste trabalho desenvolveu-se uma ferramenta numérica com acoplamento fluidomecânico (mono e bifásico) para ser utilizada em análises destes problemas na microescala (poro e grão). Utilizou-se o método dos elementos discretos (DEM) para a simulação do movimento e interação das partículas sólidas e o método de latticeBoltzmann (LBM) para a simulação do fluxo nos poros do meio geológico. A principal diferença desta ferramenta numérica em relação a trabalhos anteriores que acoplam o DEM com o LBM (DEM-LBM) está na implementação da formulação do LBM incompressível sugerida por (He e Luo, 1997) permitindo a aplicação de gradientes de pressão sensivelmente maiores do que na formulação convencional, o que é importante para as simulações de produção de sólidos. A ferramenta desenvolvida pode ser vista como um laboratório virtual para testar/verificar leis constitutivas, e que aliada a dados experimentais poderá melhorar o entendimento de mecanismos básicos envolvidos nos processos de dano mecânico de formação e de produção de sólidos. O programa computacional implementado foi verificado através de comparações com soluções analíticas ou resultados publicados na literatura. Simulações relacionadas às aplicações de interesse foram realizadas.

\section{Palavras-chave}

Método dos Elementos Discretos; Método de Lattice-Boltzmann; Dano Mecânico de Formação; Produção de Sólidos. 


\section{Abstract}

Velloso, Raquel Q.; Vargas Jr., Eurípedes do Amaral (Advisor). Numerical Analysis of Fluid Mechanical Coupling in Porous Media Using the Discrete Element Method. Rio de Janeiro, 2010. 94p. DSc Thesis - Departamento de Engenharia Civil, Pontifícia Universidade Católica do Rio de Janeiro.

The present research was mainly motivated by petroleum geomechanics problems such as solids production and formation damage. Solids production is related to phenomena whereby solid particles are produced together with fluids from reservoir rocks having little or no consolidation although it is reported that those phenomena have already happened to more resistant materials. Formation damage is the term used in order to identify permeability reduction occurring for various processes and which reduce productivity and injectivity of wells in oil and gas production systems. In the present work, a numerical tool considering fluidmechanical coupling (one and two phase flow) was developed for analyses in the microscale (pores and grains). The DEM (Discrete Element Method) was used for the simulation of motion and interaction of solid particles and the lattice Boltzmann method (LBM) for the simulation of flow inside pores of the geological media. The main difference between the developed tool and the ones developed in previous works that couple DEM with LBM is the introduction of incompressible LBM as suggested by (He e Luo, 1997), one that allows the application of pressure gradients considerably larger than the conventional formulation which is important for the simulation of solids production. The developed tool can be viewed as a virtual laboratory for testing and verification of constitutive laws which together with experimental data may improve the understanding of basic phenomena involved in formation damage and solids production. The numerical implementation was verified through comparisons with analytical solutions and other results from the literature. Simulations related to practical applications were carried out and discussed.

\section{Keywords}

Discrete Element Method; Lattice-Boltzmann Method; Formation Damage; Solid Production. 


\section{Sumário}

1 Introdução $\quad 12$

1.1 Motivação e Objetivo 12

$\begin{array}{lll}1.2 & \text { Organização } & 14\end{array}$

2 Metodologia $\quad \mathbf{1 5}$

2.1 Introdução 15

2.2 Método dos Elementos Discretos 18

2.3 Método de Lattice-Boltzmann 24

2.4 Acoplamento Fluidomecânico 38

3 Implementação $\quad 41$

4 Resultados $\quad 43$

4.1 Introdução $\quad 43$

4.2 Verificações 44

4.3 Relação Tensão - Deformação - Permeabilidade 52

4.4 Produção de Sólidos 62

$\begin{array}{lll}4.5 & \text { Força Capilar } & 72\end{array}$

$\begin{array}{lll}5 & \text { Conclusões } & 79\end{array}$

$\begin{array}{ll}\text { Referências Bibliográficas } & 82\end{array}$

A Velocidades e Coeficientes das Redes D2Q9 e D3Q19 91

B Condição de Contorno de Pressão Prescrita $\quad 93$ 


\section{Lista de Figuras}

2.1 Ciclo de cálculo do DEM 19

2.2 Representação gráfica do modelo de contato entre partículas. À esquerda na direção normal e à direita na direção cisalhante. 21

2.3 Notação usada para descrever os contatos 22

2.4 Rede D2Q9 - duas dimensões, nove velocidades 28

2.5 Domínio Periódico 31

2.6 Condição de Contorno Periódico 31

2.7 Componentes da pressão nas proximidades da interface. 35

2.8 Etapa de criação da tensão interfacial no RKLBM. As cores azul e vermelha representam as duas fases e o cinza uma combinação das duas.

2

1

31

2.9 Etapa de redistribuição das cores no RKLBM. 37

2.10 Molhabilidade dos sólidos no RKLBM. 38

2.11 Exemplo de campo de fração de sólidos 39

3.1 Esquema de acoplamento fluidomecânico 42

4.1 Perfil de velocidade - LBM convencional - $\triangle P=16.7 P a$

4.2 Perfil de velocidade - LBM incompressível - $\triangle P=16.7 \mathrm{~Pa} \quad 45$

4.3 Perfil de velocidade - LBM incompressível - $\triangle P=167 P a \quad 46$

4.4 Geometria e condições de contorno do exemplo bidimensional para a verificação da determinação da força de arraste

4.5 Comparação entre os resultados obtidos com o LBM incompressível e os resultados apresentados por Richou et al. (2004)

4.6 Comparação entre a solução analítica e a solução numérica para a força de arraste numa esfera

4.7 Condição de regime permanente para $R=0.36 \mathrm{~mm}$. O gráfico à direita mostra o perfil de pressão na reta que passa pelo centro da bolha $y=0.8 \mathrm{~mm}$.

$\begin{array}{lll}\text { 4.8 LBM bifásico - Verificação da lei de Laplace } & 49 \\ 4.9 & \text { Condição inicial para a simulação de ângulos de contatos estáticos. } & 50\end{array}$

4.10 Diferentes ângulos de contato simulados com o LBM bifásico 50

4.11 Fluxo bifásico em um tubo 3D. À esquerda o esquema da simulação. À direita a curva de permeabilidade relativa. As linhas representam a solução analítica e os pontos os resultados do LBM.

4.12 Metodologia para a obtenção das relações $\sigma \times \epsilon \times \mathrm{k}$ utilizando o DEM e o LBM

4.13 Amostra sintética e da amostra sintética (DEM) - Ensaios CTC simulação de deformação uniaxial

4.16 Estrutura porosa (em azul) utilizada nas simulações de fluxo.

4.17 Porosidade nas seções tranversais ao fluxo em cada uma das direções. 58 
4.18 Curvas tensão-deformação da amostra sintética para o ensaio CTC e os valores de permeabilidade calculados nas simulações. Os marcadores indicam os estágios onde foram realizadas as simulações de fluxo.

4.19 Curvas tensão-deformação para a simulação de deformação uniaxial e a variação da permeabilidade. Os marcadores indicam os estágios onde foram realizadas as simulações de fluxo.

4.20 Curvas de permeabilidade relativa para a amostra sintética para 2 estágios de tensão diferentes.

4.21 Distribuição dos fluidos na estrutura porosa para diversas saturações, para o estado de tensão ETO

4.22 Distribuição dos fluidos na estrutura porosa para diversas saturações, para o estado de tensão ET1

4.23 Comportamento tensão-deformação, simulado com o DEM, do material granular usado nas simulações de produção de sólidos

4.24 Região de simulação

4.25 Geometria e condição de contorno para as simulações de produção de sólidos

4.26 Curvas de produção de sólidos ( $\sigma_{c}$ em MPa, $\Delta P / L$ em $\left.\mathrm{MPa} / \mathrm{m}\right) \quad 66$

4.27 Posição das partículas. $\mathrm{t}=0$

4.28 Posição das partículas. $\mathrm{t}=0.062 \mathrm{~s}$

4.29 Posição das partículas. $\mathrm{t}=0.124 \mathrm{~s}$

68

4.30 Posição das partículas. $\mathrm{t}=0.186 \mathrm{~s}$

68

4.31 Posição das partículas. $t=0.248 \mathrm{~s}$

4.32 Cimentação entre partículas no tempo $t=0.248 \mathrm{~s}$. Os pontos azuis mostram as cimentações entre grãos que permaneceram intactas

4.33 Campo de velocidades (em $\mathrm{m} / \mathrm{s}) . \mathrm{t}=0.248 \mathrm{~s}$

4.34 Campo de pressões (em Pa). $\mathrm{t}=0.248 \mathrm{~s}$

4.35 Geometria de duas placas paralelas e o menisco entre elas.

4.36 Condição inicial para as simulações de força capilar entre placas paralelas. Os sólidos estão representados em cinza, o fluido molhante em vermelho e o fluido não molhante azul.

4.37 Condição de equilíbrio para as simulações de força capilar entre placas paralelas. Os sólidos estão representados em cinza, o fluido molhante em vermelho e o fluido não molhante em azul.

4.38 Geometria da simulação de um menisco entre duas partículas circulares.

4.39 Corte no menisco e as força atuantes. A força resultante do primeiro termo da soma é nula.

4.40 Configurações dos meniscos na condição de regime permanente. $\quad 76$

4.41 Variação da força capilar com o ângulo de molhado. A linha se refere à eq. (4.18) e os pontos aos valores obtidos numericamente.

4.42 Variação da força capilar com o deslocamento da partícula. A linha vermelha representa a força calculada através da eq. (4.18) e a linha azul o resultado da simulação com RKLBM.

A.1 Direção das velocidades discretas da rede D2Q9 91

A.2 Direção das velocidades discretas da rede D3Q19 


\section{Lista de Tabelas}

4.1 Parâmetros da simulação de fluxo entre duas placas paralelas. $\quad 44$

4.2 Parâmetros da simulação da força transferida para uma partícula sólida.

4.3 Parâmetros da simulação da lei de Laplace. 49

4.4 Dados para a contrução da amostra sintética 54

4.5 Parâmetros micromecânicos do material sintético 55

4.6 Parâmetros para as simulações de fluxo 56

4.7 Valores de permeabilidade absoluta (em darcy) 58

4.8 Parâmetros micromecânicos do material 63

4.9 Parâmetros geomecânicos macroscópicos do material 63

4.10 Parâmetros de fluxo das simulações 64

4.11 Dados para a simulação das placas paralelas. $\quad 73$

4.12 Força capilar entre duas placas paralelas. 